\title{
SACE_0012, a TetR-Family Transcriptional Regulator, Affects the Morphogenesis of Saccharopolyspora erythraea
}

\author{
Xiaojuan Yin • Xinqiang Xu $\cdot$ Hang $\mathrm{Wu} \cdot$ \\ Li Yuan · Xunduan Huang • Buchang Zhang
}

Received: 26 February 2013/Accepted: 10 May 2013/Published online: 23 June 2013

(C) The Author(s) 2013. This article is published with open access at Springerlink.com

\begin{abstract}
Saccharopolyspora erythraea, a myceliumforming actinomycete, produces a clinically important antibiotic erythromycin. Extensive investigations have provided insights into erythromycin biosynthesis in $S$. erythraea, but knowledge of its morphogenesis remains limited. By gene inactivation and complementation strategies, the TetR-family transcriptional regulator SACE_0012 was identified to be a negative regulator of mycelium formation of $S$. erythraea A226. Detected by quantitative real-time PCR, the relative transcription of $S A C E_{-} 7115$, the amfC homolog for an aerial mycelium formation protein, was dramatically increased in SACE_O012 mutant, whereas erythromycin biosynthetic gene eryA, a pleiotropic regulatory gene bldD, and the genes $S A C E \_2141$, SACE_6464, SACE_6040, that are the homologs to the sporulation regulators WhiA, WhiB, WhiG, were not differentially expressed. SACE_O012 disruption could not restore its defect of aerial development in bldD mutant, and also did not further accelerate the mycelium formation in the mutant of SACE_7040 gene, that was previously identified to be a morphogenesis repressor. Furthermore, the transcriptional level of SACE_OO12 had not markedly
\end{abstract}

Xiaojuan Yin and Xinqiang Xu contributed equally to this study.

Electronic supplementary material The online version of this article (doi:10.1007/s00284-013-0410-x) contains supplementary material, which is available to authorized users.

X. Yin $\cdot X . X u \cdot H . W u(\varangle) \cdot$ L. Yuan .

$X$. Huang $\cdot$ B. Zhang $(\square)$

Institute of Health Sciences, School of Life Sciences,

Anhui University, Jiu Long Road No. 111,

Hefei 230601, China

e-mail:wuh2007@gmail.com

B. Zhang

e-mail: zhbc@ahu.edu.cn changed in bldD and SACE_7040 mutant over A226. Taken together, these results suggest that SACE_0012 is a negative regulator of $S$. erythraea morphogenesis by mainly increasing the transcription of amfC gene, independently of the BldD regulatory system.

\section{Introduction}

During its life cycle, the soil-inhabiting Actinomycetes undergoes a complex morphological differentiation to adapt to adverse environments [1]. Growth of Actinomycetes begins with spore germination and hyphal outgrowth, leading to the formation of a vegetative, or substrate mycelium. Sensing of nutrient deprivation stimulates reproductive growth resulting in the development of aerial hyphae and spore chains [2]. Saccharopolyspora erythraea could form the aerial hyphae, and produce erythromycin, which is a macrolide antibiotic with broad-spectrum antimicrobial activity. Extensive genetic and biochemical studies have provided detailed insights into the genes involved in erythromycin biosynthesis in S. erythraea [3, 4], yet its morphological differentiation remains poorly understood.

In recent years, the availability of the complete genome sequence of $S$. erythraea allowed a deeper exploration of the molecular processes controlling its morphogenesis [5]. Guided by in vitro and in vivo investigations, BldD (SACE_2077) was discovered to be a key developmental regulator in actinomycetes [1], controlling erythromycin biosynthesis and morphological differentiation in S. erythraea [6]. Furthermore, we identified a TetR-family transcriptional regulator (SACE_7040) involving in $S$. erythraea mycelium formation, and established genetic evidence for the crosstalk between SACE_7040 and BldD 
[7]. In this study, we have used gene inactivation, complementation, and transcriptional analysis to delineate the role of a new TetR-family regulator (SACE_0012) in the development differentiation of $S$. erythraea. Deletion of $S A C E \_0012$ principally influences the transcription of a putative aerial mycelium formation gene $S A C E_{-} 7115$, that is homologous to amfC of Streptomyces.

\section{Materials and Methods}

Strains and Growth Conditions

Saccharopolyspora erythraea A226 and its derivatives were incubated in TSB medium at $30{ }^{\circ} \mathrm{C}$ for DNA extraction, protoplast preparation, and in liquid fermentation medium R5 for analysis of erythromycin production. R3M agar medium was used for protoplast regeneration, phenotypic observations, and RNA extraction [7]. Escherichia coli DH5 $\alpha$ was the host for plasmid construction [8]. Bacillus subtilis PUB110 was used for an inhibition test of erythromycin production in S. erythraea.

Plasmid, DNA Isolation, and Manipulation

Plasmid pUCTSR [9] was a pUC18 derivative containing a $1.36 \mathrm{~kb}$ fragment of a thiostrepton resistance cassette $(t s r)$ cloned into the BamHI/SmaI sites. The E. coli-S. erythraea integrative shuttle expression vector pZMW $[4,10]$ was used for the gene complementation. DNA isolation and manipulation in E. coli and S. erythraea were carried out according to the standard methods $[8,11]$.

Disruption of SACE_0012 in S. erythraea A226, bldD, and SACE_7040 Mutant

Two $1.5 \mathrm{~kb}$ fragments flanking the SACE_O012 gene were amplified from genomic DNA of S. erythraea A226 by PCR using the primer pairs P1/P2 (5'-TGC GAA TTC CTC CTC
GGC CGG TGA GCA GC-3'; 5'-GAT GGT ACC ATA CGA GCG GCC CCA ACC CGA AAG CCC- ${ }^{\prime}$ ) and P3/P4 (5'-ATT TCT AGA ACA CGC CCG CCA CCG GCT TCG C-3'; 5'-ACC AAG CTT AAG GGC TCG ATC GAC TCC TGG CGG- $3^{\prime}$ ). Then, the two DNA PCR products were inserted into the EcoRI/KpnI and XbaI/HindIII sites of pUCTSR, respectively, yielding pUCTSR $\triangle 0012$. By linearized fragment homologous recombination [7], the SACE_0012 gene was replaced with the thiostrepton resistance gene in the $S$. erythraea A226 chromosome, and the selected mutants were verified by PCR using the primers P1/ P4 (Fig. 1a, b). Similarly, SACE_0012 disruption was formed in the bldD and SACE_7040 mutant strains.

Genetic Complementation of the SACE_O012 Mutant

For complementation, the SACE_O012 gene was amplified by the primers P5 $\left(5^{\prime}\right.$-TAA CAT ATG TTG AAA ACG GCG TCA ATC CTC ATC CCG-3') and P6 (5'-CGC GAT ATC TCA GCG ATC GGC GGT AGT CG-3') from genomic DNA of $S$. erythraea A226, and was ligated into the $N d e \mathrm{I} / E c o \mathrm{RV}$ sites of pZMW [9] to generate pZMW0012. Then, pZMW-0012 was introduced into SACE_0012 mutant by PEG-mediated protoplast transformation, generating the complemented strain $\triangle S A C E \_0012 / \mathrm{pZMW}$ 0012 .

\section{Quantitative Real-Time PCR (qRT-PCR)}

The transcriptional levels of eryA, bldD, SACE_0012, and homologous genes of whiA, whiB, whiG, and amfC associated with morphogenesis in Streptomyces (Table S1) [12], were determined by qRT-PCR. Specific primers were designed as listed in Table S2. Total RNA was isolated from S. erythraea A226 and the mutants of SACE_0012, bldD, and SACE_7040 after 2 or 4 days growth on R3M agar medium. Then, extracted RNA was treated with DNase I (Fermentas), and reverse transcription was accomplished using a cDNA synthesis kit (Fermentas).
Fig. 1 Inactivation of the TetR-family regulatory gene SACE_0012. a Schematic deletion of SACE_O012 in $S$. erythraea A226. b Confirmation of SACE_0012 deletion mutant by PCR analysis with the primer pair P1/P4. The size of $3.69 \mathrm{~kb}$ for the PCR-amplified bands was observed in wild-type A226, while a band of the size $4.36 \mathrm{~kb}$ was observed in mutant $\triangle S A C E \_0012$, suggesting that the $S A C E \_0012$ gene was completely deleted a

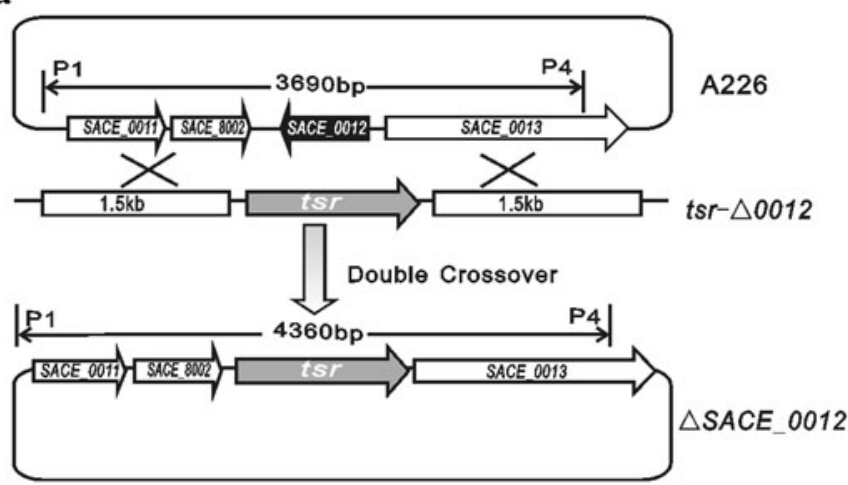

b

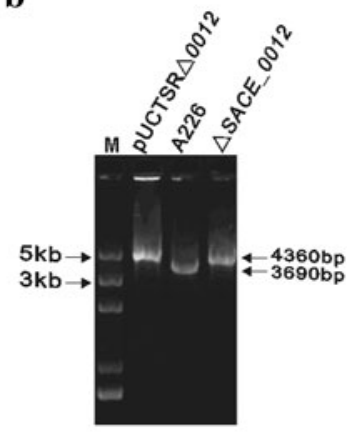


qRT-PCR reactions were performed on the Applied Biosystems StepOnePlus system with Maxima ${ }^{\text {TM }}$ SYBR Green/ROX qPCR Master Mix (Fermentas). The $h r d B$ gene encoding the major sigma factor in S. erythraea was used as an internal control, and relative quantification was evaluated using a comparative cycle threshold method as described by Livak and Schmittgen [13].

Fermentation and Analysis

Wild-type strain A226, $\triangle S A C E \_0012$, and $\triangle b l d D$ were grown in $30 \mathrm{ml}$ R5 liquid medium in $250 \mathrm{ml}$ baffled flasks for 6 days at $30{ }^{\circ} \mathrm{C}$. $5 \mu \mathrm{l}$ fermentation supernatant from these cultures was added to LB agar plates, which was sprayed with an overnight culture of B. subtilis PUB110. The plates were incubated at $37{ }^{\circ} \mathrm{C}$ for $12 \mathrm{~h}$, and the erythromycin production was estimated by scoring the growth-inhibition zones. Furthermore, erythromycin A produced by these cultures were quantitatively analyzed by high performance liquid chromatograph (HPLC) as described previously [14]. Erythromycin was isolated from the fermentation culture, and quantified by a standard curve [15].

\section{Results and Discussion}

Characterization of the SACE_0012 Gene Deletion Mutant

Given a key role of the TetR-family regulator in morphological differentiation in actinomycetes [16], by gene inactivation and phenotype observation, we have identified several TetR-family regulators related to morphogenesis in S. erythraea, including the SACE_7040 gene previously reported [7] and the SACE_0012 gene currently studied. Bioinformatic analysis shows that the $S A C E \_0012$ gene has a full-length of $690 \mathrm{bp}$ (GenBank Accession No. NC009142.114,813-115,502 nt) and is a member of the TetRfamily regulators that consists of 229 amino acids with a molecular mass of $25 \mathrm{kDa}$. To investigate its function, SACE_0012 was inactivated by replacing the 690 bp gene with a thiostrepton resistance cassette in S. erythraea A226 by the linearized fragment homologous recombination. A thiostrepton resistant mutant $\triangle S A C E \_0012$ was formed and confirmed by PCR analysis (Fig. 1a, b).

When grown on R3M medium, the mutant $\triangle S A C E \_0012$ formed aerial hypha earlier than original strain A226 in a three-day assay (Fig. 3b). When complemented with a cloned $S A C E \_0012$ under the control of the PermE* constitutive promoter (pZMW-0012), the $\triangle S A C E \_0012 / \mathrm{pZMW}-0012$ strain had restored the timing of aerial mycelium on R3M agar medium (data not shown). After a longer cultivation to the sixth day, no significant phenotypic difference was observed between the wild-type strain A226, mutant $\triangle S A C E \_0012$, and $\triangle S A C E \_0012 / \mathrm{pZME}-0012$ (data not shown), revealing that $S A C E \_0012$ was responsible for the early aerial hypha formation of S. erythraea. Moreover, $\triangle S A C E \_0012$ and A226 strains had comparable inhibition activity for B. subtilis, and produced similar amount of erythromycin A by HPLC analysis of fermentation products (Fig. S1A-B), confirming that SACE_0012 was specifically involved in the morphological differentiation of $S$. erythraea.

Effect of SACE_0012 Disruption on Transcription of the Genes for Morphogenesis and Erythromycin Biosynthesis

To test the effect of SACE_O012 disruption on the expression of morphogenesis and erythromycin biosynthesis genes, we compared A226 and mutant $\triangle S A C E \_0012$ for the transcriptional change to sporulation genes (whi and bldD), an aerial mycelium formation gene $\operatorname{amfC}$ [17], and the erythromycin structure gene eryA (Table S1). The homologous genes to whiA, whiB, whiG involved in the regulation of sporulation in Streptomyces [18] (SACE_2141, SACE_ 6464, SACE_6040, respectively) were examined by qRTPCR. SACE_2141,SACE_6464,SACE_6040 transcriptions were slightly increased but not statistically different in mutant $\triangle S A C E \_0012$ over strain A226. bldD and eryA were also not differentially expressed. However, the transcriptional levels of the amfC homolog SACE_7115 (Table S1), an aerial mycelium formation gene conserved presented in Streptomyces [17], were approximately 3.0-fold higher in the mutant $\triangle S A C E \_0012$ (Fig. 2).

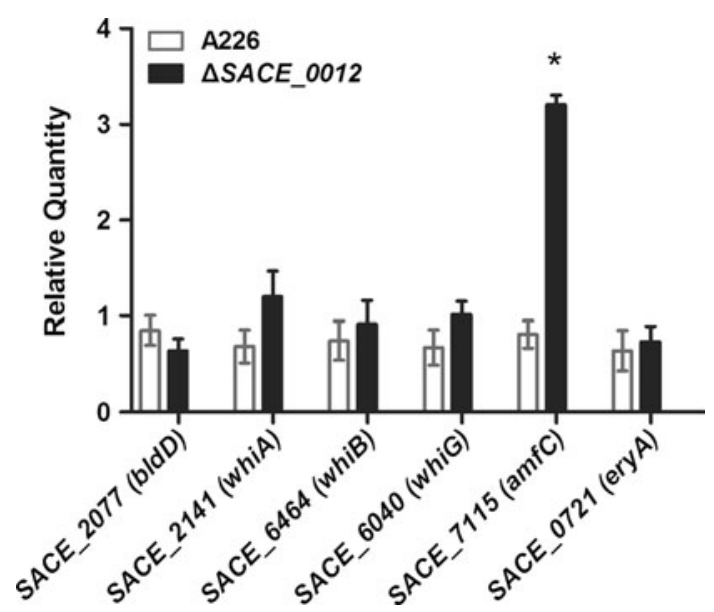

Fig. 2 Transcriptional analysis of the genes for morphogenesis and erythromycin biosynthesis in the SACE_0012 mutant. Mean values of three independent experiments are shown, with the standard deviation indicated by error bars. Statistical significance $(* P<0.05)$ compared to culture of wild-type strain A226 
Fig. 3 Confirmation and phenotype of the $\triangle b l d D /$ $\triangle S A C E \_0012$ double mutant. a Confirmation of $\triangle b l d D /$ $\triangle S A C E \_0012$ mutant by PCR analysis with the primer pair $\mathrm{P} 1 /$ P4. b Phenotype of the $\Delta b l d D /$ $\triangle S A C E \_0012$ mutant

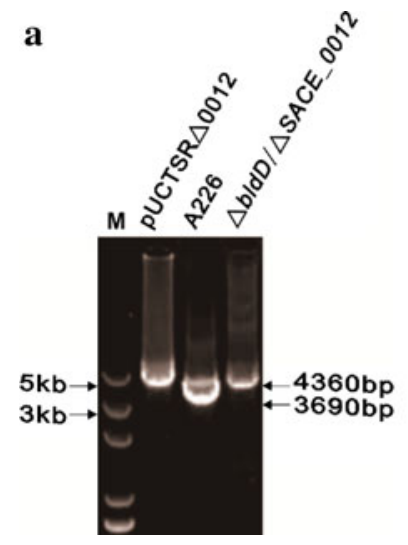

SACE_0012 Disruption Failed to Restore the Defect in the Mycelium Formation of a bldD Mutant

bldD is required for development differentiation in $S$. erythraea [1]. To examine the relationship of SACE_0012 and bldD, SACE_O012 was disrupted in the bldD mutant to form a double knockout mutant strain, $\triangle b l d D / \triangle S A C E \_0012$ (Fig. 3a). No significant phenotypic difference was observed between the mutant $\triangle$ bldD/DSACE_0012 and $\triangle b l d D$ (Fig. 3b), indicating that SACE_O012 disruption could not restore the defect of aerial hyphae in bldD mutant. qRT-PCR analysis showed that SACE_O012 transcriptions were slightly decreased but not obviously different in mutant $\triangle b l d D$ (Fig. 5). These indicate that SACE_0012, although influencing morphological differentiation, seems to have no connection with the BldD regulatory system.

\section{SACE_0012 Disruption Did Not Further Accelerate} the Mycelium Formation of SACE_7040 Mutant

We identified a TetR-family regulator SACE_7040 negatively involving in the morphological differentiation of $S$. erythraea, in which an interplay with the bldD gene was previously established [7]. Therefore, we inactivated the SACE_0012 gene in the SACE_7040 mutant to study combined effect upon the morphogenesis (Fig. 4a). It appears that the mycelium formation of SACE_7040 mutant was earlier than SACE_O012 mutant, but no obvious change of

Fig. 4 Confirmation and phenotype of the $\triangle S A C E \_7040 /$ $\triangle S A C E \_0012$ double mutant. a Confirmation of $\triangle S A C E \_7040 / \triangle S A C E \_0012$ mutant by PCR analysis with the primer pair $\mathrm{P} 1 / \mathrm{P} 4$. b Phenotype of the $\triangle S A C E \_7040 / \triangle S A C E \_0012$ mutant

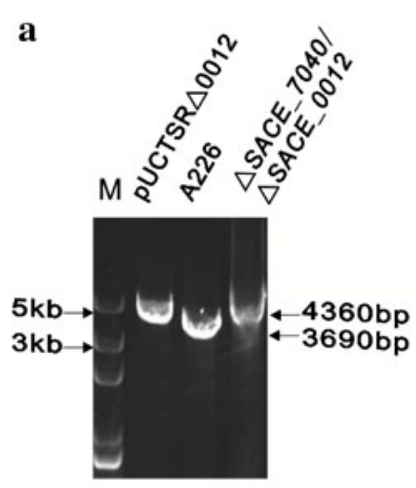

b
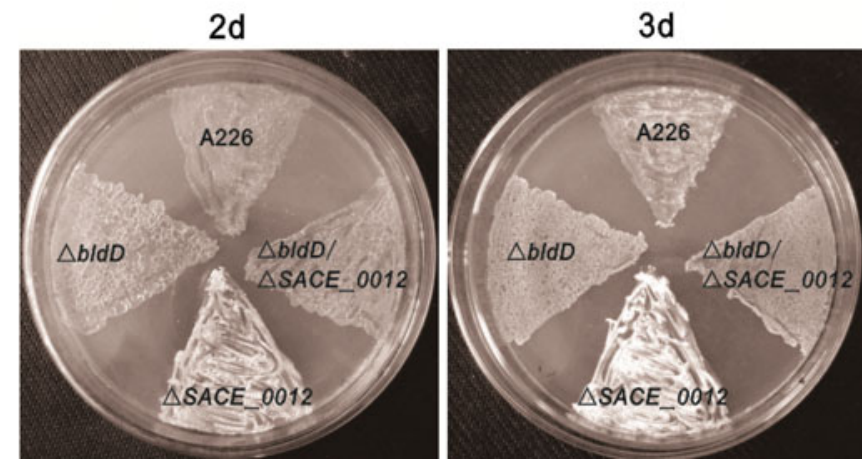

aerial mycelia was detected in the double knockout mutant $\triangle S A C E \_7040 / \triangle S A C E \_0012$ relative to the $S A C E \_7040$ mutant (Fig. 4b). Likewise, SACE_0012 transcriptions were slightly decreased but not statistically different in mutant $\triangle S A C E \_7040$ (Fig. 5).

In conclusion, these results indicated that compared with original strain A226, aerial hypha formation initiates earlier in SACE_O012 mutant. The likely cause of an early aerial hypha formation is the higher transcriptional level of $a m f C$. Previous genetic evidences revealed that amfC positively controlled aerial mycelium formation in Streptomyces coelicolor and Streptomyces griseus, and was distributed widely in this genus [17], implying that amfC might be under the control of $S A C E \_0012$ to affect the early aerial hypha formation of $S$. erythraea. In addition, we found that SACE_OO12 disruption could not restore the defect of aerial development in a bldD mutant, and also could not further accelerate the mycelium formation in a mutant of $S A C E \_7040$ gene. Further qRT-PCR analysis showed that $S A C E \_0012$ transcriptions were slightly decreased but not obviously different in mutant $\triangle b l d D$ and $\triangle S A C E \_7040$ relative to A226. Thus, SACE_0012 was likely independent of the BldD regulatory system for controlling $S$. erythraea morphogenesis, distinct from the TetR-family regulator $S A C E \_7040$ previously reported [7].

With structural and sequence conserved analysis [19], homologous of SACE_OO12 are predominantly distributed in rare actinomycetes, such as Amir_6428 from Actinosynnema mirum (identities $50 \%$ ), BN6_77090 from Saccharothrix

b

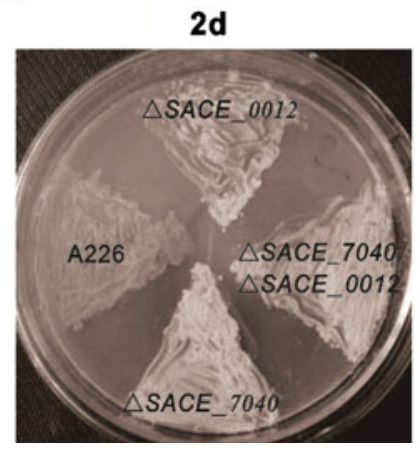




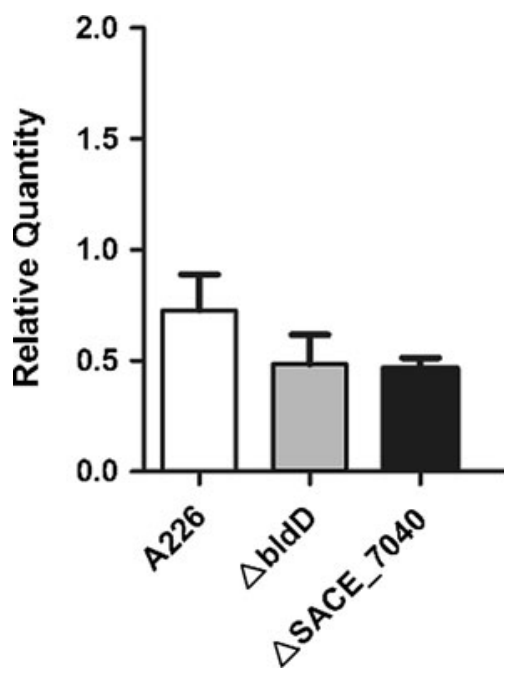

Fig. 5 Relative transcriptional levels of SACE_O012 in the deletion mutants of bldD and SACE_7040 over A226. Mean values of three independent experiments are shown, with the standard deviation indicated by error bars

espanaensis (identities $50 \%$ ), AMED_0889 from Amycolatopsis mediterranei (identities $48 \%$ ), etc. (Fig. S3). However, functional analysis of the TetR-family regulator has been never reported, signifying a new regulatory mechanism for mycelial formation in actinomycetes, such as how it works with its ligand and target [10]. Therefore, these findings provide novel insights into $S$. erythraea developmental biology. In the future, more detailed regulatory mechanism of the SACE_O012 gene will likely be valuable to deepening the understanding of the modulation of $S$. erythraea morphogenesis.

Acknowledgments We are grateful to Professor Yiguang Wang (Chinese Academy of Medical Sciences, Beijing, China) for providing S. erythraea A226 and Professor David T. Weaver (Anhui University, Hefei, China) for reviewing the manuscript. This study was supported by Grants from the National Basic Research Program (973) (Grant No. 2013CB734001), the National Natural Science Foundation of China (Grant Nos. 30870069) and The Natural Science Foundation of Anhui Province (Grant No. 1208085MC46).

Open Access This article is distributed under the terms of the Creative Commons Attribution License which permits any use, distribution, and reproduction in any medium, provided the original author(s) and the source are credited.

\section{References}

1. Elliot M, Damji F, Passantino R et al (1998) The bldD gene of Streptomyces coelicolor A3(2): a regulatory gene involved in morphogenesis and antibiotic production. J Bacteriol 180(6): $1549-1555$

2. Kelemen GH, Buttner MJ (1998) Initiation of aerial mycelium formation in Streptomyces. Curr Opin Microbiol 1:656-662

3. Cane DE (2010) Programming of erythromycin biosynthesis by a modular polypeptide synthase. J Biol Chem 285(36):2751727523

4. Weber JM, Leung JO, Maine GT et al (1990) Organization of a cluster of erythromycin genes in Saccharopolyspora erythraea. J Bacteriol 172:2372-2383

5. Oliynyk M, Samborskyy M, Lester JB et al (2007) Complete genome sequence of the erythromycin-producing bacterium Saccharopolyspora erythraea NRRL23338. Nat Biotechnol 25(4):447-453

6. Chng C, Lum AM, Vroom JA et al (2008) A key developmental regulator controls the synthesis of the antibiotic erythromycin in Saccharopolyspora erythraea. PNAS 105(32):11346-33351

7. Han S, Song P, Ren T et al (2011) Identification of SACE_7040, a member of TetR family related to the morphological differentiation of Saccharopolyspora erythraea. Curr Microbiol 63(2): 121-125

8. Sambrook J, Russell DW (2001) Molecular cloning: a laboratory manual. Cold Spring Harbor Laboratory, New York

9. Zhang BC, Li LL, Yu XQ et al (2003) Construction of Saccharopolyspora erythraea expression vector pZMW. Bull Acad Mil Med Sci 27(3):176-179

10. Corre $C$ (2013) In search of the missing ligands for TetR family regulators. Chem Biol 20(2):140-142

11. Bierman M, Logan R, O’Brien K et al (1992) Plasmid cloning vectors for the conjugal transfer of DNA from Escherichia coli to Streptomyces spp. Gene 116(1):43-49

12. McCormick JR, Flärdh K (2012) Signals and regulators that govern Streptomyces development. FEMS Microbiol Rev 36(1): 206-231

13. Livak KJ, Schmittgen TD (2001) Analysis of relative gene expression data using real-time quantitative PCR and the $2^{-\Delta \Delta \mathrm{CT}}$. Method 25(4):402-408

14. Tsuji K, Goetz JF (1978) HPLC as a rapid means of monitoring erythromycin and tetracycline fermentation processes. J Antibiot (Tokyo) 31(4):302-308

15. Wu J, Zhang Q, Deng W et al (2011) Toward improvement of erythromycin A production in an industrial Saccharopolyspora erythraea strain via facilitation of genetic manipulation with an artificial attB site for specific recombination. Appl Environ Microbiol 77(21):7508-7516

16. Ramos JL, Martinez-Bueno M, Molina-Henares AJ et al (2005) The TetR family of transcriptional repressors. Microbiol Mol Biol Rev 69(2):326-356

17. Yonekawa T, Ohnishi Y, Horinouchi S et al (1999) Involvement of amfC in physiological and morphological development in Streptomyces coelicolor A3(2). Microbiology 145:2273-2280

18. Flardh K, Findlay KC, Chater KF et al (1999) Association of early sporulation genes with suggested developmental decision points in Streptomyces coelicolor A3(2). Microbiology 145: 2229-2243

19. Yu Z, Reichheld SE, Savchenko A et al (2010) A comprehensive analysis of structural and sequence conservation in the TetR family transcriptional regulators. J Mol Biol 23(4):847-864 